\title{
Enhanced plumbagin production in Plumbago indica root culture by simultaneous and sequential dual elicitations using chitosan with L-alanine and methyl- $\beta$-cyclodextrin
}

\author{
Amit Jaisi ${ }^{1}$ and Pharkphoom Panichayupakaranant ${ }^{2,3^{*}}$
}

\begin{abstract}
The simultaneous and sequential dual elicitation effect on plumbagin production in Plumbago indica L. root cultures, revealed that combination of chitosan $\left(150 \mathrm{mg} \mathrm{L}^{-1}\right)$ with $\mathbf{L}$-alanine $(5 \mathrm{mM})$ or methyl- $\beta$-cyclodextrin (MCD; $\left.2 \mathrm{mM}\right)$ significantly increased plumbagin production, but in the different treatment manners. The simultaneous treatment using chitosan + L-alanine on a 14-day-old culture enhanced plumbagin production to $14.62 \mathrm{mg} \mathrm{g}^{-1} \mathrm{DW}$, while the sequential additions of MCD to a 12-day-old culture followed by chitosan after $48 \mathrm{~h}$ enhanced production of plumbagin to $14.33 \mathrm{mg} \mathrm{g}^{-1} \mathrm{DW}$. The plumbagin productivity from both treatments were up to 1.3- and 8-fold higher than the chitosan treated (10.93 $\left.\mathrm{mg} \mathrm{g}^{-1} \mathrm{DW}\right)$ and untreated root cultures $\left(1.76 \mathrm{mg} \mathrm{g}^{-1} \mathrm{DW}\right)$. Moreover, the present studies provided new information on the effect of simultaneous and sequential elicitation on plumbagin-producing $P$. indica root cultures using chitosan in combinations with MCD or L-alanine.
\end{abstract}

Keywords: L-Alanine, Chitosan, Dual elicitation, Methyl- $\beta$-cyclodextrin, Plumbagin, Plumbago indica

\section{Introduction}

Plumbagin (5-hydroxy-2-methyl-1,4-naphthoquinone), a bioactive constituent in various medicinal products, and most prominently present in Plumbago species especially the roots of Plumbago indica L. (Plumbaginaceae) (Mallavadhani et al. 2002). The demand for the large-scale production of plumbagin has gained tremendous focus due to its attractive pharmacological effects (Kaewbumrung and Panichayupakaranant 2012), and low supply cause of low yields from traditional route to fill the gap (Komaraiah et al. 2003). Moreover, unfeasibility of plumbagin chemical synthesis, and incomplete information of plumbagin biosynthesis restricts application of metabolic engineering for its production. Presently, application of

\footnotetext{
*Correspondence: pharkphoom.p@psu.ac.th

2 Phytomedicine and Pharmaceutical Biotechnology Excellence Center, Faculty of Pharmaceutical Sciences, Prince of Songkla University, Hat-Yai, Songkhla 90112, Thailand

Full list of author information is available at the end of the article
}

elicitors to plant cell/organ cultures has been found as one of the most effective biotechnological approaches to induce or enhance the production of plumbagin in $P$. indica.

A wide variety of elicitors such as yeast extracts (Putalun et al. 2010; Juengwatanatrakul et al. 2011), chitosan (Komaraiah et al. 2003; Gangopadhyay et al. 2011; Jaisi and Panichayupakaranant 2016a, 2017), methyl jasmonate (Gangopadhyay et al. 2011; Martin et al. 2011), salicylic acid (Silja et al. 2014) and gamma ray irradiation (Jaisi et al. 2013) have been employed to induce the biosynthesis of plumbagin in various plant cell/organ cultures of plumbagin-producing plants. Recently, $P$. indica root cultures have been employed to enhance plumbagin production using different biotic and abiotic elicitors (Panichayupakaranant and Tewtrakul 2002; Jaisi et al. 2013; Jaisi and Panichayupakaranant 2016a, b, c, 2017). In addition, our recent works demonstrated that chitosan $\left(150 \mathrm{mg} \mathrm{L}^{-1}\right)$ is the most suitable elicitor for enhanced 
production of plumbagin in $P$. indica root cultures (Jaisi and Panichayupakaranant 2016a, b, 2017).

However, till date most researchers have focused on the effect of an individual elicitor for plumbagin productivity. Apparently, different elicitors have different mode of actions, and the elicitors depending on different signal transductions induce different proteins and secondary metabolites (Cho et al. 2008). These plant signal transduction cascades involved in secondary metabolites production is a complicated network. Lately, researchers have observed that the combined use of two different elicitors can be more effective due to a synergistic or additive or potentiating effect (Zhao et al. 2001; Pourianezhad et al. 2019). One such strategy is the combination of a biotic or abiotic elicitor with one of the signal molecules in plant defense responses such as salicylic acid or methyl jasmonate. For example, 55-fold increased taxane production in Taxus $x$ media cell cultures were achieved using cyclodextrin and methyl jasmonate (Sabater-Jara et al. 2014). Nonetheless, even application of two different elicitors was also found effective, for instance 17- to 40-fold enhanced trans-resveratrol production in Vitis vinifera L. suspension culture cells using cyclodextrins and coronatine (Almagro et al. 2015). Moreover, the application of different elicitors at suitable time intervals has enhanced secondary metabolite production more than the treatments in the same period. Such sequential treatment has been reported to be effective strategy. Cho et al. (2008) have reported that the production of benzophenanthridine alkaloids in Eschscholzia californica Cham. suspension cultures through sequential treatments with methyl jasmonate, salicylic acid and yeast extracts was more effective than simultaneous or single treatments. In addition, $24 \mathrm{~h}$ prior treatment of Salvia miltiorrhiza Bunge. hairy root cultures with methyl jasmonate potentiated the yeast extract-induced tanshinone production (Ge and Wu 2005). Similarly, dual elicitation using low concentration of $\mathrm{Ag}^{+}$and $\mathrm{Cd}^{+}$stimulated phenolic acid accumulation in $V$. vinifera cell suspension cultures without loss of cell viability (Cai et al. 2013). Selection of efficient elicitors for synergistic combination and period for the treatment are therefore the most important and practical ways to improve secondary metabolites production.

Notably, there has been so far no report concerning simultaneous or sequential dual combination effects on $P$. indica root cultures. Based on our previous characterization of suitable biotic and abiotic elicitors (Jaisi and Panichayupakaranant 2016a), further focus on an improved plumbagin production using the dual combination of chitosan and a particular elicitor, i.e., methyl jasmonate, salicylic acid, yeast extracts, 1-naphthol, $\mathrm{MCD}, \mathrm{AgNO}_{3}$, sodium acetate and L-alanine was performed. The effects of simultaneous and sequential treatments of the dual combined elicitors on production of plumbagin and root biomass were also determined.

\section{Materials and methods Root cultures}

The root cultures of $P$. indica were established and maintained in the cultured conditions as described previously by Jaisi and Panichayupakaranant (2016a). Briefly, the root cultures were initiated from the young leaf explants, and maintained in 250-mL Erlenmeyer flasks containing $50 \mathrm{~mL}$ Gamborg's B5 liquid medium supplemented with $0.1 \mathrm{mg} \mathrm{NAA} \mathrm{L}^{-1}$ and $20 \mathrm{~g}$ sucrose $\mathrm{L}^{-1}$, on a rotary shaker $(60 \mathrm{rpm})$, at $25 \pm 2{ }^{\circ} \mathrm{C}$ under dark conditions. The root cultures ( $2 \mathrm{~g}$ fresh weight) were maintained in the same conditions by a 3 -week periodic subculture.

\section{Elicitor preparation}

Different type and concentration of elicitors, i.e., methyl jasmonate $(50 \mu \mathrm{M})$, salicylic acid $(50 \mu \mathrm{M})$, yeast extracts $\left(100 \mathrm{mg} \mathrm{L}^{-1}\right), \mathrm{MCD}(2 \mathrm{mM}), \mathrm{AgNO}_{3}(100 \mu \mathrm{M})$, 1-naphthol $(50 \mu \mathrm{M})$, sodium acetate $(5 \mathrm{mM})$, and L-alanine (5 mM), were selected to combine with chitosan (150 mg L ${ }^{-1}$ ) according to our previous study (Jaisi and Panichayupakaranant 2016a). Yeast extract was purified by ethanol precipitation as described by Hahn and Albersheim (1978) before use. The elicitor stock solutions were prepared as follows; methyl jasmonate, salicylic acid, and 1-naphthol were prepared in $40 \% \mathrm{v} / \mathrm{v}$ ethanol, while yeast extracts, chitosan, $\mathrm{MCD}, \mathrm{AgNO}_{3}$, sodium acetate and $\mathrm{L}$-alanine were prepared in distilled water. The yeast extract and chitosan stock solutions were autoclaved for 15 min at $121{ }^{\circ} \mathrm{C}$, while the other elicitor stock solutions were filtered through a microfilter $(0.20 \mu \mathrm{m})$ and stored at $4{ }^{\circ} \mathrm{C}$ until use.

\section{Simultaneous and sequential elicitation}

Elicitor treatment combination, time for sequential addition of elicitors and harvest time was selected according to biomass and plumbagin content reported in our previous study (Jaisi and Panichayupakaranant 2016a, c, 2017). For a simultaneous treatment, the 14-day-old root cultures were simultaneously added with any combination of chitosan and a particular elicitor. For a sequential treatment, the 12-day-old root cultures were added with chitosan, and after $48 \mathrm{~h}$ followed by an addition of a particular elicitor. The sequential treatment was also performed vice versa, started with a particular elicitor and followed by chitosan. The same amount of water or ethanol was added to the control cultures throughout the experiments.

After the treatments, the root cultures were continually cultured in normal culture conditions for another 
$72 \mathrm{~h}$, and then the root biomass was harvested from the culture medium by a vacuum filtration and washed with distilled water to remove residual medium. The roots were dried at $50{ }^{\circ} \mathrm{C}$ for $24 \mathrm{~h}$. The dry weight (DW) of roots was recorded using an analytical balance and stored at $-20{ }^{\circ} \mathrm{C}$ until use. The harvested cultured medium was also frozen at $-20^{\circ} \mathrm{C}$ until use.

\section{Quantitative plumbagin analysis}

Quantitative determination of plumbagin was performed using the HPLC method as described previously by Jaisi et al. (2013). In brief, the dried root powders $(100 \mathrm{mg})$ were extracted with ethanol $(20 \mathrm{~mL})$ under reflux conditions for $20 \mathrm{~min}$, and then filtered. The cultured medium $(50 \mathrm{~mL})$ was acidified with $1 \mathrm{~N}$ $\mathrm{HCl}$ to $\mathrm{pH} 2.0$, and then extracted with ethyl acetate $(20 \mathrm{~mL} \times 3)$. The treated resin was extracted with methanol $(20 \mathrm{~mL} \times 3)$ on a shaker $(60 \mathrm{rpm})$, for $12 \mathrm{~h}$. The obtained extracts were evaporated under reduced pressure at $40{ }^{\circ} \mathrm{C}$, and the residue was reconstituted in methanol, and the volume adjusted to $10 \mathrm{~mL}$.

The analysis was performed by HPLC (Waters 1525, Westbrook, CT) system, set up using a C18 column (Phenomenex ${ }^{\circledR}$ ODS, $5 \mu \mathrm{m}, 150 \mathrm{~mm} \times 4.6 \mathrm{~mm}$ i.d.). The mobile phase was a mixture of methanol and $5 \%$ aqueous acetic acid $(80: 20, \mathrm{v} / \mathrm{v})$, with an isocratic elution at a flow rate of $0.85 \mathrm{~mL} \mathrm{~min}{ }^{-1}$. The quantitative detection wavelength was set at $260 \mathrm{~nm}$. The content of plumbagin was calculated by comparing with the standard curve of authentic plumbagin (Sigma-Aldrich, USA). The experiments were performed in triplicate.

\section{Data processing and analysis}

The plumbagin content and the total plumbagin yield ( $\mathrm{mg} \mathrm{L}^{-1}$ media) were used to determine any effects of the elicitations. The plumbagin content was calculated by combining the intracellular and extracellular content of plumbagin based on a dry biomass $\left(\mathrm{mg} \mathrm{g}^{-1} \mathrm{DW}\right)$. The total yield of plumbagin was calculated by multiplying the plumbagin content with the dry weight of root biomass per 1-L media $\left(\mathrm{mg} \mathrm{L}^{-1}\right)$.

All experiments were performed in minimum triplicate $(n=3)$ and values regarding the biomass, plumbagin content and total plumbagin yield are presented as a mean $\pm \mathrm{SE}$. The values were analyzed using oneway ANOVA followed by Tukey's multiple range tests, and the differences at $p \leq 0.05$ were regarded as significant.

\section{Results and discussion}

\section{Effects of simultaneous elicitation on plumbagin} production

Our present study indicated that only a combination of chitosan and L-alanine improved the plumbagin production in the simultaneous dual elicitation. The production of plumbagin was up to $14.62 \mathrm{mg} \mathrm{g}^{-1} \mathrm{DW}$ that was 1.43 -fold higher than the chitosan single treatment (10.93 $\mathrm{mg} \mathrm{g}^{-1}$ DW) (Fig. 1a). Some of the other simultaneous dual elicitations did influence the plumbagin production, but was significantly lower than the single chitosan elicitation (Fig. 1). In addition, regarding the dual elicitation effect on growth of the root culture, the total yields of plumbagin production were calculated based on the cultured root biomass in $\mathrm{mg} \mathrm{L}^{-1}$. The chitosan and $\mathrm{L}$-alanine simultaneous treatment provoked the highest total plumbagin yield of $43.87 \mathrm{mg} \mathrm{L}^{-1}$ that was up to 1.67 -fold higher than the chitosan single-elicited group (26.22 mg $\mathrm{L}^{-1}$ ) (Fig. 1b). Such magnitude of an enhanced plumbagin production using the dual simultaneous elicitation with chitosan and L-alanine could be due to the possible role of $\mathrm{L}$-alanine as a precursor for plumbagin biosynthesis as reported by Rischer et al. (2002), along with the effects of chitosan inducing defense responses (Iriti and Faoro 2009), cell permeability effects (Johnson et al. 1991), or chitosan might have influenced on the enzymes involved in the biosynthesis of plumbagin like that of phenylpropaniod pathway (Chakraborty et al. 2009). Similarly, dual combination effect on plumbagin production has been reported through a simultaneous methyl jasmonate and chitosan treatment in the hairy root culture of $P$. indica (Gangopadhyay et al. 2011). Notably, the dual elicitation with chitosan and L-alanine or MCD did not affect the dried root biomass compared to the untreated group (Fig. 1c).

In addition, the root cultures simultaneously treated with chitosan and MCD significantly improved the intracellular plumbagin production up to $3.87 \mathrm{mg} \mathrm{g}^{-1}$ that was 2.2- and 2.85-fold higher than the control (1.76 $\left.\mathrm{mg} \mathrm{g}^{-1} \mathrm{DW}\right)$ and chitosan-alone treated groups $\left(1.36 \mathrm{mg} \mathrm{g}^{-1} \mathrm{DW}\right)$. It is in accordance to the higher intracellular accumulation of anthraquinone in suspension cultures of Rubia tinctorum L. by combined treatment using cyclodextrins and methyl jasmonate (Perassolo et al. 2016) and parthenolide in cell suspension of Tanacetum parthenium by combined treatment of yeast extracts and methyl jasmonate (Pourianezhad et al. 2019). The productivity obtained through this key strategy, was much higher than those previously reported in the chitosan-treated root cultures of $P$. indica (10.6 $\mathrm{mg} \mathrm{g}^{-1} \mathrm{DW}$ ) (Jaisi and Panichayupakaranant 2016a). Also comparatively higher than the yeast extract treated in vitro cultures of Drosera indica L. (2.69 $\mathrm{mg} \mathrm{g}^{-1} \mathrm{DW}$ ) (Juengwatanatrakul et al. 

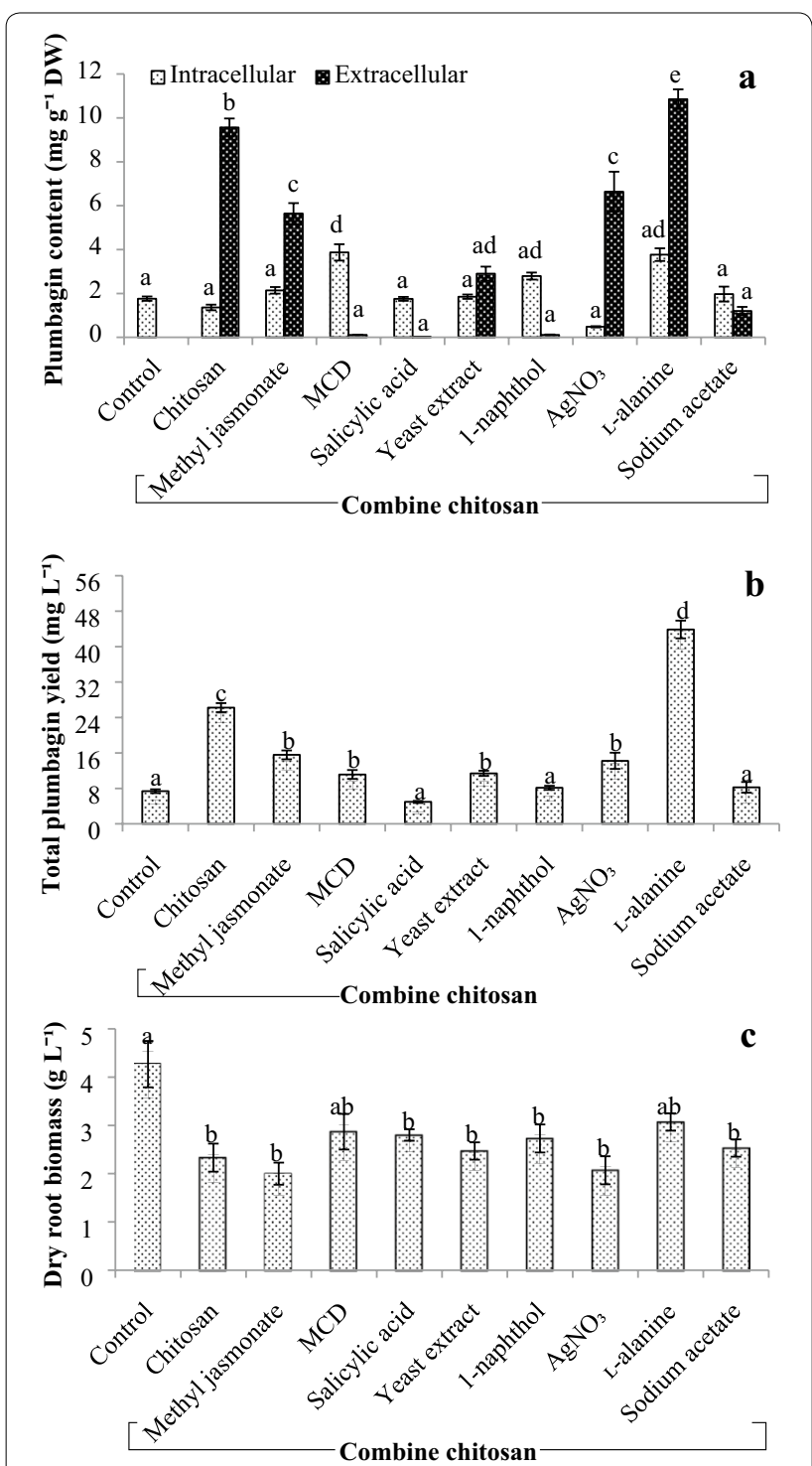

Fig. 1 Effect of simultaneous treatments using chitosan and other elicitors on a plumbagin content; $\mathbf{b}$ total plumbagin yield; $\mathbf{c}$ dry root biomass. Fourteen-day $P$. indica root cultures were treated with $150 \mathrm{mg}$ chitosan $\mathrm{L}^{-1}$ (singly) or combined with methyl jasmonate, salicylic acid, 1-naphthol, yeast extract, $\mathrm{MCD}, \mathrm{AgNO}_{3}$, sodium acetate and $\mathbf{L}$-alanine. Control is untreated root cultures with equal amount of distilled water or $2 \%$ ethanol added on the 14 th day. Measurements were taken after 72-h treatment from day 14 treatments. Values are mean \pm SE of triplicate experiments. Data were analyzed by one-way ANOVA, using Tukey's test for multiple comparison. Different superscripts letters between the groups are significantly different at $p<0.05$. MCD; methyl- $\beta$-cyclodextrin

2011), and D. burmannii (8.8 $\mathrm{mg} \mathrm{g}^{-1} \mathrm{DW}$ ) (Putalun et al. 2010), and the combined methyl jasmonate and chitosan-treated P. indica hairy root cultures in a bioreactor system (13.16 $\mathrm{mg} \mathrm{g}^{-1} \mathrm{DW}$ ) (Gangopadhyay et al. 2011).
The present study demonstrated that the combined elicitation using either chitosan and 1-naphthol or chitosan and sodium acetate insignificantly reduced plumbagin production when compared to each of the single treatment (Jaisi and Panichayupakaranant 2016a). Similar reports on lower production in simultaneous dual combination of methyl jasmonate and chitosan compared to methyl jasmonate single treatments was observed in betacyanin accumulation in Portulaca sp. cv. cell suspension cultures (Bhuiyan and Adachi 2003). These results indicated that simultaneous dual elicitations may not only give a synergistic effect, but also an antergic effect on secondary metabolite productions. However, the order of the dual treatments may also play an important role in their effects on secondary metabolite production. A sequential treatment strategy for further improved plumbagin production was therefore further studied.

\section{Effects of sequential elicitation on plumbagin production}

The sequential dual elicitation strategy using a 48-h prior chitosan treatment followed by a treatment with a particular elicitor did not significantly improve the plumbagin productivity compared to the single chitosan treatment group. Although the sequential treatments of chitosan followed by methyl jasmonate gave the highest total plumbagin content $\left(15.08 \mathrm{mg} \mathrm{g}^{-1} \mathrm{DW}\right)$ that was 1.38-fold higher than the chitosan-alone treated groups (10.93 $\left.\mathrm{mg} \mathrm{g}^{-1} \mathrm{DW}\right)$ (Fig. 2a), no significant difference in their total plumbagin yields (chitosan; $26.22 \mathrm{mg} \mathrm{L}^{-1}$ and chitosan + methyl jasmonate; $30.16 \mathrm{mg} \mathrm{L}^{-1}$ ) was observed (Fig. 2b). However, a significant decrease in the root biomass was observed in all sequential treatments, except for the chitosan combinations with L-alanine or MCD when compared with those of the control group (Fig. 2c). Noteworthy, most sequential treatments lead to higher extracellular released plumbagin contents than those of the simultaneous treatments, which might have affected the dry root biomass, especially through the phytotoxic action of plumbagin (Gonçalves et al. 2009).

Moreover, the effects of vice versa sequential treatments for the dual combined elicitors were determined (Fig. 3). The key combined elicitors that enhanced plumbagin production through this dual sequential treatment were MCD followed by chitosan, with the total plumbagin content of $14.33 \mathrm{mg} \mathrm{g}^{-1} \mathrm{DW}$ that was 1.3-fold higher than chitosan single treatment (10.93 $\left.\mathrm{mg} \mathrm{g}^{-1} \mathrm{DW}\right)$ (Fig. 3a). In addition, its total plumbagin yield ( $\left.40.12 \mathrm{mg} \mathrm{L}^{-1}\right)$ was also significantly higher than that of the chitosan single treated group $(26.22 \mathrm{mg}$ $\mathrm{L}^{-1}$ ) (Fig. 3b). This may be due to the effect of MCD on induction of plant defense responses. It has been reported that cyclodextrins have been shown to trigger a signal transduction cascade involved in secondary 

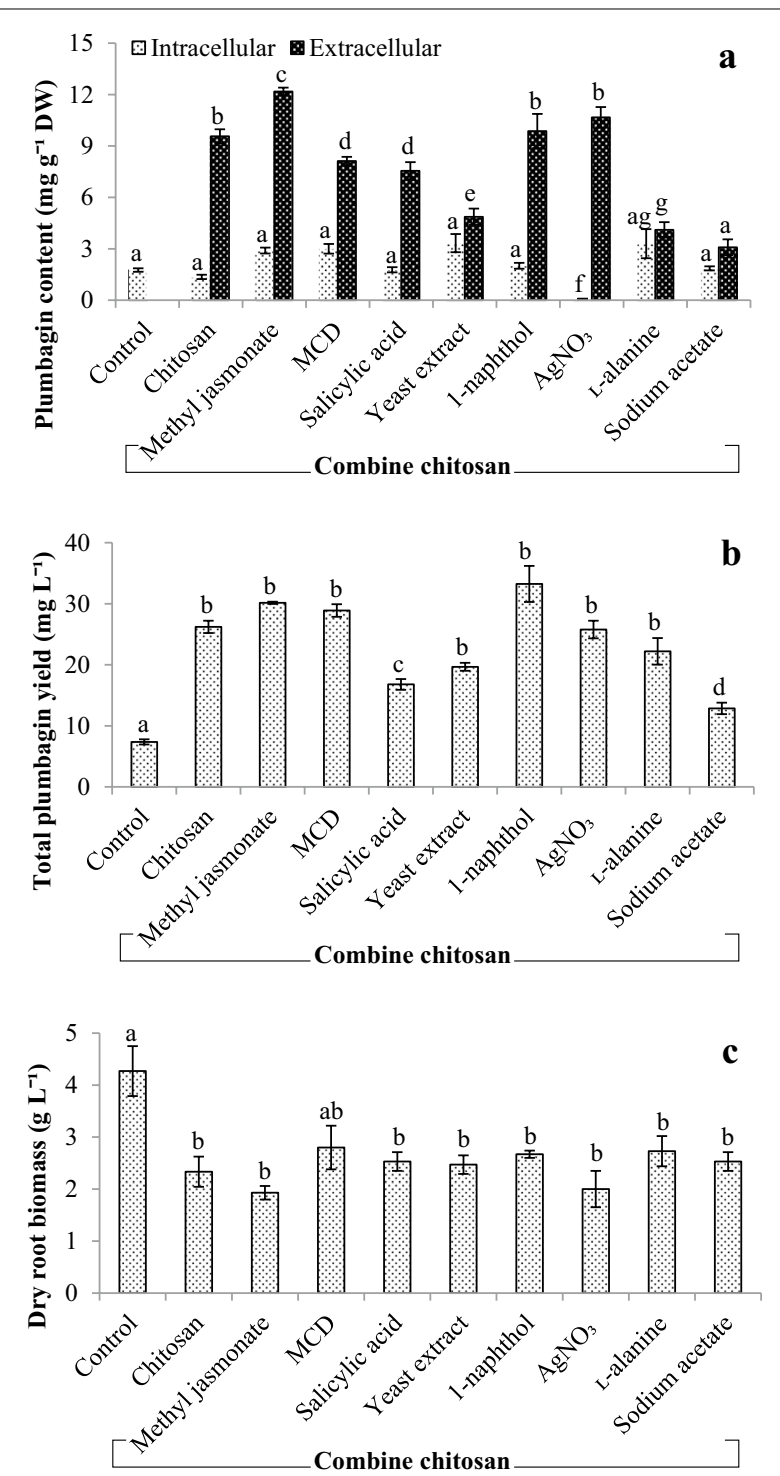

Fig. 2 Effect of sequential treatments using chitosan followed by other elicitors on a plumbagin content; $\mathbf{b}$ total plumbagin yield; $\mathbf{c}$ dry root biomass. Twelve-day P. indica root cultures were treated with $150 \mathrm{mg} \mathrm{L}^{-1}$ chitosan followed by 14-day treatments with methyl jasmonate, salicylic acid, 1-naphthol, yeast extract, MCD, $\mathrm{AgNO}_{3}$, sodium acetate and $\mathbf{L}$-alanine. Control is untreated root cultures with equal amount of distilled water or $2 \%$ ethanol added. Chitosan is the singly treated at 14 days. Measurements were taken after 72 -h treatment from day 14 treatments. Values are mean \pm SE of triplicate experiments. Data were analyzed by one-way ANOVA, using Tukey's test for multiple comparison. Different superscripts letters between the groups are significantly different at $p<0.05$. MCD; methyl- $\beta$-cyclodextrin

metabolites pathway, resulting in enhanced production of taxane in Taxus $x$ media cell cultures using cyclodextrin and methyl jasmonate (Sabater-Jara et al. 2014). Interestingly, only the sequential treatment using $\mathrm{MCD}$ followed by chitosan showed no significant different in
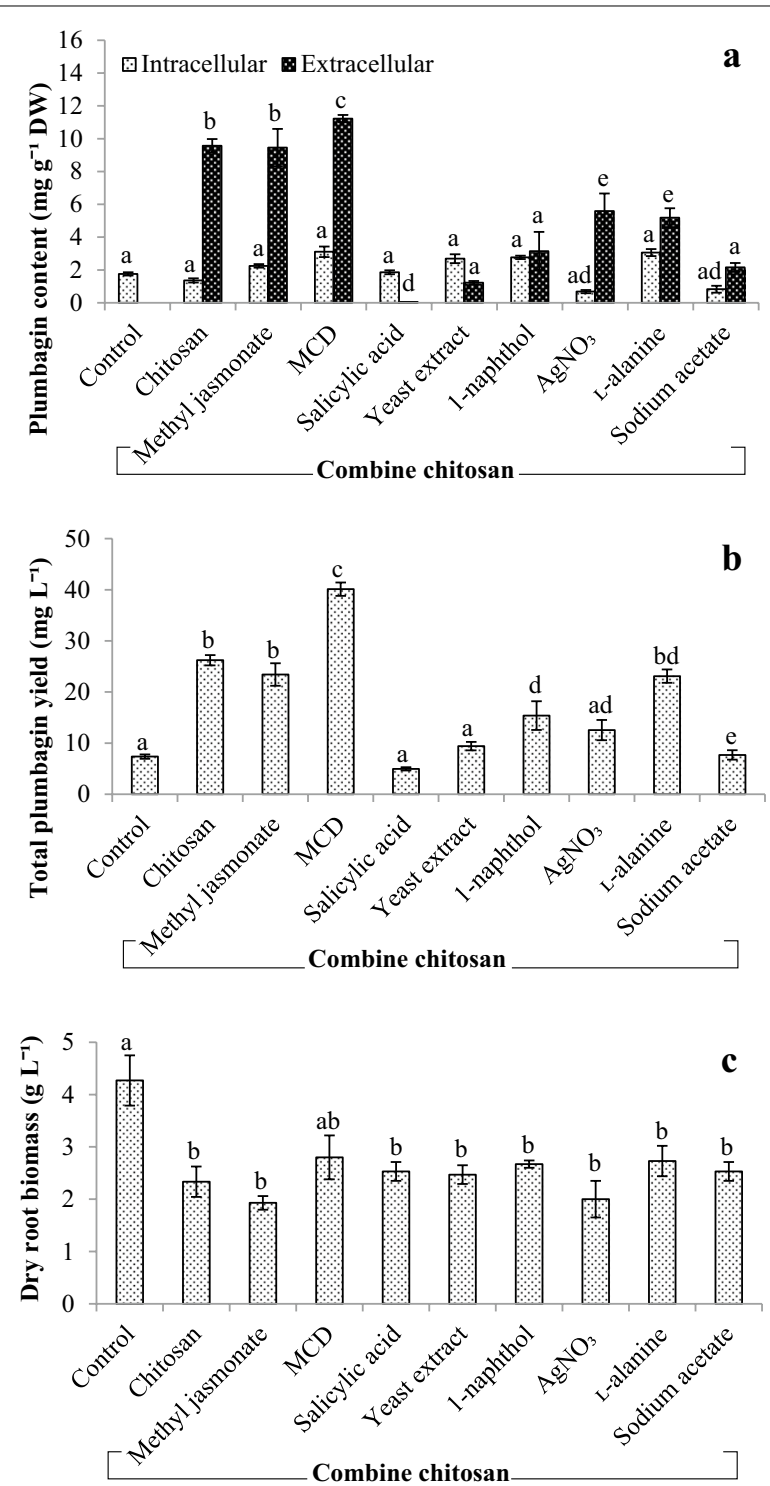

Fig. 3 Effect of sequential treatments using other elicitors followed by chitosan on a plumbagin content; $\mathbf{b}$ total plumbagin yield; $\mathbf{c}$ dry root biomass. Twelve-day $P$. indica root cultures were treated with methyl jasmonate, salicylic acid, 1-naphthol, yeast extract, $\mathrm{MCD}, \mathrm{AgNO}_{3}$, sodium acetate and $\mathrm{L}$-alanine, then followed by $150 \mathrm{mg} \mathrm{L}^{-1}$ chitosan at 14 days. Control is untreated root cultures with equal amount of distilled water or $2 \%$ ethanol added. Chitosan is the singly treated at 14 days. Measurements were taken after $72 \mathrm{~h}$ treatment from day 14 treatments. Values are mean \pm SE of triplicate experiments. Data were analyzed by one-way ANOVA, using Tukey's test for multiple comparison. Different superscripts letters between the groups are significantly different at $p<0.05$. MCD; methyl- $\beta$-cyclodextrin

the root biomass when compared to the control group (Fig. 3c). This result implied that MCD may reduce phytotoxicity of chitosan and plumbagin via its inclusion complex formations with chitosan and plumbagin. 
The simultaneous or sequential treatment of chitosan in dual combination with a particular elicitor accelerated plumbagin production by diverse ways; the mechanism for every treatment may be more intricate since it depends on the interactions between physiological effects caused by two treatments as observed by Zhao et al. (2005). In insectivorous plants, plumbagin is restricted to the traps suggesting its possible role as defense mechanism against microbes brought by visiting prey and additionally act as molecular triggers in prey capture and digestion (Galek et al. 1990; Raj et al. 2011; Widhalm and Rhodes 2016). Raj et al. (2011) reported that in Nepenthes khasiana, chitin induces production of droserone and 5-O-methyl droserone. Similarly, chitin induces the production of plumbagin in Drosophyllum lusitanicum suspension cultures (Nahálka et al. 1998). Such phenomenon of plumbagin increase due to chitosan was also observed in in vitro culture of D. indica (Juengwatanatrakul et al. 2011) and D. burmannii (Putalun et al. 2010). It is likely that the induced plumbagin production in these studies could be due to mimicking of natural defense mechanism (Iriti and Faoro 2009) or might have triggered the expression of enzymes involved in plumbagin biosynthesis similarly to other plant secondary metabolites (Chakraborty et al. 2009; Baque et al. 2012). Till date, signaling mechanism of elicitors influencing plumbagin production or complete plumbagin biosynthesis is largely unknown.

It is interesting to note the order of treatments using chitosan and a particular elicitor plays an important role on the enhanced plumbagin production. The variability of plumbagin production observed in dual elicitation of chitosan with plant signaling molecules like salicylic acid or methyl jasmonate might be due to inhibition of the degree of acetylation, a mechanism by which chitosan induces the defense responses (Xing et al. 2015) or might be the cross-interference between the two distant pathways of salicylic acid and jasmonic acid signal transduction or the electrostatic interactions. Similar observation is reported on crossinterference or negative interaction between the plant signaling molecules and combining elicitors (salicylic acid or methyl jasmonate) (Vidal et al. 1997; Schweizer et al. 1997; Bhuiyan and Adachi 2003). In both elicitations, the order of sequential treatments with chitosan combining 1-naphthol, $\mathrm{AgNO}_{3}$, salicylic acid, yeast extract and sodium acetate did not enhance the plumbagin production compared to single chitosan-treated group (Figs. 1, 2 and 3). Both strategies, simultaneous and sequential combination of chitosan with L-alanine or MCD favored plumbagin production with insignificant effect on root dry weight.

\section{Conclusion}

In conclusion, the present study revealed that plumbagin production considerably depends on the type of elicitor combination, elicitor contact period and growth stage of the root culture for treatment. The key success strategies include, the simultaneous treatments with chitosan $\left(150 \mathrm{mg} \mathrm{L}^{-1}\right)$ and $\mathrm{L}$-alanine $(5 \mathrm{mM})$ on the 14-day-old root culture for $72 \mathrm{~h}$, and the sequential treatments with MCD (2 mM) on the 12-day-old root culture followed the chitosan treatment after $48 \mathrm{~h}$. These two methods gave a total plumbagin production of 14.62 - and 14.33- $\mathrm{mg} \mathrm{g}^{-1} \mathrm{DW}$, respectively, within 17 days of culture. These culture systems will be useful in designing an intensive approach to improve plumbagin production and the root biomass through in situ adsorption techniques that are feasible for large-scale production of plumbagin. Moreover, the present studies provided new information on the effect of simultaneous and sequential elicitation effects on plumbagin-producing $P$. indica root cultures using chitosan in combination with MCD, L-alanine and 1-naphthol. Notably, such synergistic strategies on $P$. indica root cultures could be a useful model-system to study the transcriptomic and metabolic profiles for better knowledge and understanding on plumbagin biosynthetic pathway underlying the mechanism for the enhanced production through elicitation.

\section{Abbreviations}

DW: Dry weight; MCD: Methyl- $\beta$-cyclodextrin; NAA: 1-Naphthalene acetic acid.

\section{Acknowledgements}

The authors wish to thank Dr. Brian Hodgson for assistance with the English. This work was partially supported by the new strategic research (CGS-

P2P-2562-049) project, Walailak University, Thailand.

\section{Authors' contributions}

AJ and PP conceived and designed the research. AJ conducted the experiments. AJ and PP analyzed data and wrote manuscripts. Both authors read and approved the final manuscript.

\section{Funding}

This work was supported by Prince of Songkla University, Thailand.

\section{Availability of data and materials}

All data generated or analyzed during this study are included in this article.

Ethics approval and consent to participate

Not applicable.

\section{Consent for publication}

Not application.

\section{Competing interests}

The authors declare that they have no competing interests.

\section{Author details}

${ }^{1}$ School of Pharmacy, Walailak University, Thasala, Nakhon Si Tham-

marat 80160, Thailand. 2 Phytomedicine and Pharmaceutical Biotechnology Excellence Center, Faculty of Pharmaceutical Sciences, Prince of Songkla 
University, Hat-Yai, Songkhla 90112, Thailand. ${ }^{3}$ Department of Pharmacognosy and Pharmaceutical Botany, Faculty of Pharmaceutical Sciences, Prince of Songkla University, Hat-Yai, Songkhla 90112, Thailand.

Received: 3 December 2019 Accepted: 17 February 2020

Published online: 28 February 2020

\section{References}

Almagro L, Belchí-Navarro S, Martínez-Márquez A, Bru R, Pedreño MA (2015) Enhanced extracellular production of trans-resveratrol in Vitis vinifera suspension cultured cells by using cyclodextrins and coronatine. Plant Physiol Biochem 97:361-367

Baque MA, Shiragi MH, Lee EJ, Paek KY (2012) Elicitor effect of chitosan and pectin on the biosynthesis of anthraquinones, phenolics and flavonoids in adventitious root suspension cultures of Morinda citrifolia (L.). Aust J Crop Sci 6:1349-1355

Bhuiyan MNH, Adachi T (2003) Stimulation of betacyanin synthesis through exogenous methyl jasmonate and other elicitors in suspension-cultured cells of Portulaca. J Plant Physiol 160:1117-1124

Cai Z, Kastell A, Speiser C, Smetanska I (2013) Enhanced resveratrol production in Vitis vinifera cell suspension cultures by heavy metals without loss of cell viability. Appl Biochem Biotechnol 171:330-340

Chakraborty M, Karun A, Mitra A (2009) Accumulation of phenylpropanoid derivatives in chitosan-induced cell suspension culture of Cocos nucifera. J Plant Physiol 166:63-71

Cho HY, Son SY, Rhee HS, Yoon SYH, Lee-Parsons CW, Park JM (2008) Synergistic effects of sequential treatment with methyl jasmonate, salicylic acid and yeast extract on benzophenanthridine alkaloid accumulation and protein expression in Eschscholtzia californica suspension cultures. J Biotechnol 135:117-122

Galek H, Osswald WF, Elstner EF (1990) Oxidative protein modification as predigestive mechanism of the carnivorous plant Dionaea muscipula: an hypothesis based on in vitro experiments. Free Radic Biol Med 9:427-434

Gangopadhyay M, Dewanjee S, Bhattacharya S (2011) Enhanced plumbagin production in elicited Plumbago indica hairy root cultures. J Biosci Bioeng 111:706-710

Ge X, Wu J (2005) Induction and potentiation of diterpenoid tanshinone accumulation in Salvia miltiorrhiza hairy roots by $\beta$-aminobutyric acid. Appl Microbiol Biotechnol 68:183-188

Gonçalves S, Ferraz M, Romano A (2009) Phytotoxic properties of Drosophyllum lusitanicum leaf extracts and its main compound plumbagin. Sci Hort 122:96-101

Hahn MG, Albersheim P (1978) Host-pathogen interactions. XIV. Isolation and partial characterization of an elicitor from yeast extract. Plant Physiol 62:107-111

Iriti M, Faoro F (2009) Chitosan as a MAMP, searching for a PRR. Plant Signal Behav 4:66-68

Jaisi A, Sakunphueak A, Panichayupakaranant P (2013) Increased production of plumbagin in Plumbago indica root cultures by gamma ray irradiation. Pharm Biol 51:1047-1051

Jaisi A, Panichayupakaranant P (2016a) Increased production of plumbagin in Plumbago indica root cultures by biotic and abiotic elicitors. Biotechnol Lett 38:351-355

Jaisi A, Panichayupakaranant P (2016b) Simultaneous heat shock and in situ adsorption enhance plumbagin production in Plumbago indica root cultures. Eng Life Sci 16:417-423

Jaisi A, Panichayupakaranant P (2016c) Enhanced plumbagin production in Plumbago indica root cultures by $\mathrm{L}$-alanine feeding and in situ adsorption. Plant Cell Tiss Org Cult 1:53-60

Jaisi A, Panichayupakaranant P (2017) Chitosan elicitation and sequential Diaion ${ }^{\circledR} \mathrm{HP}-20$ addition a powerful approach for enhanced plumbagin production in Plumbago indica root cultures. Process Biochem 53:210-215

Johnson TS, Ravishankar GA, Venkataraman LV (1991) Elicitation of capsaicin production in freely suspended cells and immobilized cell cultures of Capsicum frutescens mill. Food Biotechnol 5:197-205
Juengwatanatrakul T, Sakamoto S, Tanaka H, Putalun W (2011) Elicitation effect on production of plumbagin in in vitro culture of Drosera indica L. J Med Plants Res 5:4949-4953

Kaewbumrung S, Panichayupakaranant P (2012) Isolation of three antibacterial naphthoquinones from Plumbago indica roots and development of a validated quantitative HPLC analytical method. Nat Prod Res 26:2020-2023

Komaraiah P, Ramakrishna SV, Reddanna P, Kavi Kishor PB (2003) Enhanced production of plumbagin in immobilized cells of Plumbago rosea by elicitation and in situ adsorption. J Biotechnol 101:181-187

Mallavadhani UV, Sahu G, Muralidhar J (2002) Screening of Plumbago species for the bio-active marker plumbagin. Pharm Biol 40:508-511

Martin KP, Sabovljevic A, Madassery J (2011) High-frequency transgenic plant regeneration and plumbagin production through methyl jasmonate elicitation from hairy roots of Plumbago indica L. J Crop Sci Biotech 14:205-212

Nahálka J, Nahálková J, Gemeiner P, Blanárik P (1998) Elicitation of plumbagin by chitin and its release into the medium in Drosophyllum lusitanicum Link. suspension cultures. Biotechnol Lett 20:841-845

Panichayupakaranant P, Tewtrakul S (2002) Plumbagin production by root cultures of Plumbago rosea. Electron J Biotechnol 5:228-232

Perassolo M, Smith ME, Giulietti AM, Talou JR (2016) Synergistic effect of methyl jasmonate and cyclodextrins on anthraquinone accumulation in cell suspension cultures of Morinda citrifolia and Rubia tinctorum. Plant Cell Tiss Org Cult 124:319-330

Pourianezhad F, Rahnama H, Mousavi A, Khosrowshahli M, Mafakheri S (2019) Parthenolide production in cell suspension culture of feverfew. Bioresour Bioprocess 6:23

Putalun W, Udomsin O, Yusakul G, Juengwatanatrakul T, Sakamoto S, Tanaka H (2010) Enhanced plumbagin production from in vitro cultures of Drosera burmannii using elicitation. Biotechnol Lett 32:721-724

Raj G, Kurup R, Hussain AA, Baby S (2011) Distribution of naphthoquinones, plumbagin, droserone, and 5-O-methyl droserone in chitin-induced and uninduced Nepenthes khasiana: molecular events in prey capture. J Exp Bot 62:5429-5436

Rischer H, Hamm A, Bringmann G (2002) Nepenthes insignis uses a C 2-portion of the carbon skeleton of $\mathrm{L}$-alanine acquired via its carnivorous organs, to build up the allelochemical plumbagin. Phytochemistry 59:603-609

Sabater-Jara AB, Onrubia M, Moyano E, Bonfill M, Palazón J, Pedreño MA, Cusidó RM (2014) Synergistic effect of cyclodextrins and methyl jasmonate on taxane production in Taxus $x$ media cell cultures. Plant Biotechnol J 12:1075-1084

Schweizer P, Buchala A, Silverman P, Seskar M, Raskin I, Metraux J-P (1997) Jasmonate-inducible genes are activated in rice by pathogen attack without a concomitant increase in endogenous jasmonic acid levels. Plant Physiol 114:79-88

Silja PK, Gisha GP, Satheeshkumar K (2014) Enhanced plumbagin accumulation in embryogenic cell suspension cultures of Plumbago rosea L. following elicitation. Plant Cell Tiss Org Cult 119:469-477

Vidal S, Leon I, Denecke J, Palva ET (1997) Salicylic acid and the plant pathogen Erwinia carotovora induce defense genes via antagonistic pathways. Plant J 11:115-123

Widhalm JR, Rhodes D (2016) Biosynthesis and molecular actions of specialized 1, 4-naphthoquinone natural products produced by horticultural plants. Hort Res. 3:1-7

Xing K, Zhu X, Peng X, Qin S (2015) Chitosan antimicrobial and eliciting properties for pest control in agriculture: a review. Agron Sustain Dev 35:569-588

Zhao J, Zhu W, Hu Q (2001) Effects of stress factors, bioregulators and synthetic precursor on indole alkaloid production in compact callus clusters cultures of Catharanthus roseus. Appl Microbiol Biotechnol 55:693-698

Zhao J, Davis LC, Verpoorte R (2005) Elicitor signal transduction leading to production of plant secondary metabolites. Biotechnol Adv 23:283-333

\section{Publisher's Note}

Springer Nature remains neutral with regard to jurisdictional claims in published maps and institutional affiliations. 